\title{
A Laurent series proof of the Habsieger-Kadell $q$-Morris identity
}

\author{
Guoce Xin \\ Department of mathematics \\ Capital Normal University \\ Beijing 100048, P.R. China \\ guoce.xin@gmail.com
}

\author{
Yue Zhou* \\ School of Mathematics and Statistics \\ Central South University \\ Changsha 410075, P.R. China \\ nkzhouyue@gmail.com
}

Submitted: Mar 24, 2014; Accepted: Sep 9, 2014; Published: Sep 18, 2014

Mathematics Subject Classifications: 05A30, 33D70

\begin{abstract}
We give a Laurent series proof of the Habsieger-Kadell $q$-Morris identity, which is a common generalization of the $q$-Morris identity and the Aomoto constant term identity. The proof allows us to extend the theorem for some additional parameter cases.
\end{abstract}

Keywords: Laurent series; constant term identities; $q$-Morris identity; $q$-Dyson identity; Selberg integral

\section{Introduction}

This paper is closely related to the well-known Dyson's ex-conjecture. The conjecture was made by Freeman Dyson in 1962 when studying statistical theory of energy levels of complex systems [7].

Theorem 1.1. For nonnegative integers $a_{0}, \ldots, a_{n}$,

$$
\mathrm{CT} \prod_{0 \leqslant i<j \leqslant n}\left(1-\frac{x_{i}}{x_{j}}\right)^{a_{i}}\left(1-\frac{x_{j}}{x_{i}}\right)^{a_{j}}=\frac{\left(a_{0}+\cdots+a_{n}\right) !}{a_{0} ! a_{1} ! \cdots a_{n} !},
$$

where $\mathrm{CT}_{\mathbf{x}} f(\mathbf{x})$ means to take the constant term in the Laurent expansion of $f(\mathbf{x})$ in the powers of $x_{0}, x_{1}, \ldots, x_{n}$.

*Corresponding author 
Dyson's ex-conjecture has been proved by many authors using different methods. See, e.g., [8, 10, 11, 21, 22]. Many variations of Dyson's ex-conjecture have been found, such as the famous Macdonald constant term conjectures $[6,17]$. Some of them are still not solved. See, e.g., $[4,5]$.

The $q$-analogous of the Dyson conjecture was made by Andrews [1] in 1975.

Theorem 1.2 (Zeilberger-Bressoud). For nonnegative integers $a_{0}, a_{1}, \ldots, a_{n}$,

$$
\mathrm{CT} \prod_{0 \leqslant i<j \leqslant n}\left(\frac{x_{i}}{x_{j}}\right)_{a_{i}}\left(\frac{x_{j}}{x_{i}} q\right)_{a_{j}}=\frac{(q)_{a_{0}+a_{1}+\cdots+a_{n}}}{(q)_{a_{0}}(q)_{a_{1}} \cdots(q)_{a_{n}}},
$$

where $(z)_{m}:=\frac{(z ; q)_{\infty}}{\left(z q^{m} ; q\right)_{\infty}}=(1-z)(1-z q) \cdots\left(1-z q^{m-1}\right)$.

Almost all methods for Dyson's ex-conjecture fail to extend for the $q$ version. Up to now, only three different methods succeeded: the combinatorial proof in [23], the short proof in [9] using iterated Laurent series, and the one page proof in [14] using the Combinatorial Nullstellensatz. The methods apply to some constant terms of similar type.

In this paper we study the Habsieger-Kadell $q$-Morris identity, an important variation of the equal parameter case of the $q$-Dyson theorem. The original identity studies the constant term of the following Laurent polynomial for $m+l \leqslant n$ :

$$
\begin{aligned}
& A_{q}\left(x_{0}, x_{1}, \ldots, x_{n} ; a, b, k, m, l\right) \\
& \quad=\prod_{i=1}^{n}\left(\frac{q^{\chi(i \leqslant m)} x_{0}}{x_{i}}\right)_{a-\chi(i \leqslant m)}\left(\frac{q^{\chi(i>m)} x_{i}}{x_{0}}\right)_{b+\chi(i \leqslant m)+\chi(i \geqslant n-l+1)} \prod_{1 \leqslant i<j \leqslant n}\left(\frac{x_{i}}{x_{j}}\right)_{k}\left(\frac{x_{j}}{x_{i}} q\right)_{k},
\end{aligned}
$$

where the expression $\chi(S)$ is 1 if the statement $S$ is true, and 0 otherwise.

In giving a Laurent series proof of the Habsieger-Kadell $q$-Morris identity, we are able to establish a unified formula that also works for the additional cases $m+l>n$. The result is stated as follows, where the additional boldfaced part $\chi(\mathbf{i} \geqslant \mathbf{2 n}-\mathbf{m}-\mathbf{l})$ is only effective when $m+l>n$.

Theorem 1.3. For nonnegative integers $a, b, k, m, l$ satisfying $m, l<n$, we have

$$
\mathrm{CT}_{x} A_{q}\left(x_{0}, x_{1}, \ldots, x_{n} ; a, b, k, m, l\right)=M_{n}(a, b, k, m, l ; q),
$$

where

$$
M_{n}(a, b, k, m, l ; q)=\prod_{i=0}^{n-1} \frac{(q)_{a+b+i k+\chi(i \geqslant n-l)}(q)_{(i+1) k}}{(q)_{a+i k-\chi(i<m)}(q)_{b+i k+\chi(i \geqslant n-m-l)+\chi(\mathbf{i} \geqslant \mathbf{2 n - m}-\mathbf{l})}(q)_{k}} .
$$

The $m=l=0, q=1$ case of the result is the Morris identity, which is equivalent to the well-known Selberg integral [19]. In his thesis [18] Morris established the identity and conjectured the $q$-analogous identity. The $q$-Morris identity, or the $m=l=0$ case, was proved by Habsieger [12] and later by Zeilberger [24]. The $m=0, q=1$ case of the 
result, called the Aomoto identity, was constructed by Aomoto [3]. By extending Aomoto's method Kadell [13] constructed the $m+l \leqslant n$ case, in the same year of Habsieger's proof. As far as we know, the $m+l>n$ case was not considered before.

Our approach is by extending the proof of the Aomoto identity in [8]. The basic idea is to regard both sides of (1.3) as polynomials in $q^{a}$ of degree at most $d=n b+m+l$. Then to show the equality of the two polynomials, it is sufficient to show that they are equal at $d+1$ points. Note that this basic idea was used by Habsieger for $q$-Selberg integral in [12]. The equality at the $d$ vanishing points are not hard to handle by the techniques in $[9,16]$. But in this approach, we have to deal with two problems: i) the multiple roots problem for small $k$; ii) the $d+1$-st suitable point is hard to find. We handle the former problem by a rationality result of Stembridge, and the latter problem by a hard searching process.

We present the major steps of our proof in Section 2. The steps are expanded by the rationality result in Section 3, by the proof of the vanishing lemma in Section 4, and by the proof for the extra point in Section 5.

While we were finishing the presented work, the one page proof of the $q$-Dyson theorem was published. Moreover, Károlyi and Nagy [15] gave a generalization of Theorem 1.3 in the $m=0$ case using the Combinatorial Nullstellensatz. The two approaches are different but have some connections.

\section{Proof of the Habsieger-Kadell $q$-Morris identity}

Following notations in the introduction, we may assume that $0 \leqslant m, l<n$ by the following argument. If $m \geqslant n$ then

$$
A_{q}\left(x_{0}, \ldots, x_{n} ; a, b, k, m, l\right)=\prod_{i=1}^{n}\left(\frac{q x_{0}}{x_{i}}\right)_{a-1}\left(\frac{x_{i}}{x_{0}}\right)_{b+1+\chi(i \geqslant n-l+1)} \prod_{1 \leqslant i<j \leqslant n}\left(\frac{x_{i}}{x_{j}}\right)_{k}\left(\frac{x_{j}}{x_{i}} q\right)_{k},
$$

which is just $A_{q}\left(x_{0} q, \ldots, x_{n} ; a-1, b+1, k, 0, l\right)$. Then by substituting $x_{0}$ by $x_{0} / q$, we can see that the constant term is $M_{n}(a-1, b+1, k, 0, l ; q)$. The case $l \geqslant n$ is similar: we observe that $A_{q}\left(x_{0}, \ldots, x_{n} ; a, b, k, m, l\right)$ can be rewritten as $A_{q}\left(x_{0}, \ldots, x_{n} ; a, b+1, k, m, 0\right)$.

Let us rewrite $M\left(q^{a}, q^{k}\right)=M_{n}(a, b, k, m, l ; q)$ as

$$
\begin{aligned}
M\left(q^{a}, q^{k}\right)= & \frac{(q)_{n k}}{(q)_{k}^{n}} \cdot \prod_{i=0}^{m-1}\left(1-q^{a+i k}\right) \cdot \prod_{i=n-l}^{n-1}\left(1-q^{a+i k+b+1}\right) \\
& \cdot \prod_{i=0}^{n-1} \frac{\left(1-q^{a+i k+1}\right)\left(1-q^{a+i k+2}\right) \cdots\left(1-q^{a+i k+b}\right)(q)_{i k}}{(q)_{b+i k+\chi(i \geqslant n-m-l)+\chi(i \geqslant 2 n-m-l)}} .
\end{aligned}
$$

We have the following characterization.

Lemma 2.1. For fixed $b, n \in \mathbb{N}$ and $0 \leqslant m, l<n, M\left(q^{a}, q^{k}\right)$ is uniquely determined by the following three properties. 
1. $M\left(q^{a}, q^{k}\right)(q)_{k}^{n} /(q)_{n k}$ is a polynomial in $q^{a}$ of degree $n b+m+l$, whose coefficients are rational functions in $q^{k}$ and $q$.

2. For any $k>b+1, M\left(q^{-\xi}, q^{k}\right)=0$ if $\xi$ belongs to one of the following three sets:

$$
\begin{aligned}
& D_{1}=\{0, k, \ldots,(m-1) k\} ; \\
& D_{2}=\{(n-l) k+b+1,(n-l+1) k+b+1 \ldots,(n-1) k+b+1\} ; \\
& D_{3}=\{i k+1, i k+2 \ldots, i k+b \mid i=0, \ldots, n-1\} .
\end{aligned}
$$

3. For any $k>b+1$ we have $M\left(q^{-(n-l-1) k-b-1}, q^{k}\right)=M_{n}(-(n-l-1) k-b-$ $1, b, k, m, l ; q)$.

Proof. Assume $M^{\prime}\left(q^{a}, q^{k}\right)$ also satisfies the above three properties. Then for every $\xi \in$ $D_{i}, i=1,2,3$ or $\xi=(n-l-1) k+b+1$,

$$
M\left(q^{-\xi}, q^{k}\right)(q)_{k}^{n} /(q)_{n k}=M^{\prime}\left(q^{-\xi}, q^{k}\right)(q)_{k}^{n} /(q)_{n k}, \quad \text { for all } k>b+1 .
$$

Since both sides are rational functions in $q^{k}$ and they agree at infinitely many points, they are identical as rational functions.

Now as polynomials in $q^{a}$, whose coefficients are rational functions in $q^{k}$ and $q$, $M\left(q^{a}, q^{k}\right)(q)_{k}^{n} /(q)_{n k}$ agrees with $M^{\prime}\left(q^{a}, q^{k}\right)(q)_{k}^{n} /(q)_{n k}$ at $n b+m+l+1$ distinct $\xi$ 's as above, they must be equal to each other.

Note that the condition $k>b+1$ can not be dropped, since $D_{3}$ has duplicate elements when $k \leqslant b-1$, and $D_{3}$ or $D_{2}$ intersects $D_{1}$ if $k=b$ or $k=b+1$.

Denote by $M_{n}^{\prime}(a, b, k, m, l ; q)$ the left-hand-side of (1.3). Then Theorem 1.3 will follow by induction on $n$ if we can show the following three lemmas, whose proofs will be given in later sections.

Lemma 2.2. For fixed $b, n \in \mathbb{N}$ and $0 \leqslant m, l<n, M_{n}^{\prime}(a, b, k, m, l ; q)(q)_{k}^{n} /(q)_{n k}$ is a polynomial in $q^{a}$ of degree at most $n b+m+l$, whose coefficients are rational functions in $q^{k}$ and $q$.

Since $M_{n}^{\prime}(a, b, k, m, l ; q)$ is a polynomial in $q^{a}$, the definition of $a$ can be extended for all integers, in particular for negative integers $a$.

Lemma 2.3 (Vanishing Lemma). For fixed $b, n \in \mathbb{N}$, and $0 \leqslant m, l<n$, and $k>b+1$, $M_{n}^{\prime}(-h, b, k, m, l ; q)$ vanishes when $h$ equals one of the values in $(2.2)$.

Remark 2.4. At this stage we can already claim the truth of Theorem 1.3 for $m=$ 0 . In this case $D_{1}$ is empty, so that we can choose $a=0$ as the extra point. Then $M_{n}^{\prime}(0, b, k, 0, l ; q)$ reduces to $M_{n}^{\prime}(0,0, k, 0,0 ; q)$, and the equal parameter case of the $q$ Dyson theorem applies.

Lemma 2.5. For fixed $b, n \in \mathbb{N}$, and $0 \leqslant m, l<n$, and $k>b+1$, if we assume Theorem 1.3 holds for smaller values of $n$, then $M_{n}^{\prime}(-h, b, k, m, l ; q)=M_{n}(-h, b, k, m, l ; q)$ when $h=(n-l-1) k+b+1$.

The extra point in the above lemma is found through a hard searching process. It is a surprise for this special $h$ : the constant term $M_{n}^{\prime}(-h, b, k, m, l ; q)$ reduces to a single constant term that can be evaluated by Remark 2.4 or the hypothesis. 


\section{The polynomial-rational characterization}

To prove Lemma 2.2, we need the the following rationality result, which is implicitly due to J.R. Stembridge [20], as can be seen from the proof. The $q=1$ case of this result is the equal parameter case of [8, Proposition 2.4].

Proposition 3.1. For any $n \in \mathbb{N}$ and $\alpha=\left(\alpha_{1}, \ldots, \alpha_{n}\right) \in \mathbb{Z}^{n}$ with $\sum_{1 \leqslant i \leqslant n} \alpha_{i}=0$, we have

$$
\left[x^{\alpha}\right] \prod_{1 \leqslant i<j \leqslant n}\left(\frac{x_{i}}{x_{j}}\right)_{k}\left(\frac{x_{j}}{x_{i}} q\right)_{k}=\frac{(q)_{n k}}{(q)_{k}^{n}} \cdot R_{n}\left(q^{k}, q ; \alpha\right) .
$$

where $R_{n}\left(q^{k}, q ; \alpha\right)$ is a rational function in $q^{k}$ and $q$, and $\left[x^{\alpha}\right]$ refers to take the coefficient of $x_{1}^{\alpha_{1}} \cdots x_{n}^{\alpha_{n}}$ in the polynomial.

Proof. In [20, Equation 44] Stembridge gave the following equation (set $z=q^{k}$ )

$$
\left[x^{\alpha}\right] \prod_{1 \leqslant i<j \leqslant n}\left(\frac{x_{i}}{x_{j}}\right)_{k}\left(\frac{x_{j}}{x_{i}} q\right)_{k}=\frac{1}{(q)_{k-1}^{n}} \sum_{S} \pm\left(-q^{k}\right)^{|S|} C^{n}[S]\left(q^{k}, q\right),
$$

where the summation is taken over some elements whose number is bounded by a function of $n$ and $C^{n}[S]\left(q^{k}, q\right)$ is a formal power series in $q^{k}$ and $q$.

By [20, Corollary 3.3] we know that

$$
C^{n}[\varnothing]\left(q^{k}, q\right)=\frac{(q)_{n k}}{\left(1-q^{k}\right)\left(1-q^{2 k}\right) \cdots\left(1-q^{n k}\right)} .
$$

In [20, Page 334, Line 33] Stembridge stated that $C^{n}[\lambda]\left(q^{k}, q\right)$ is of the form $f_{\lambda}\left(q^{k}, q\right)$. $C^{n}[\varnothing]\left(q^{k}, q\right)$ for some rational function $f_{\lambda}$. Therefore, combining with (3.2) and (3.3) we get

$$
\left[x^{\alpha}\right] \prod_{1 \leqslant i<j \leqslant n}\left(\frac{x_{i}}{x_{j}}\right)_{k}\left(\frac{x_{j}}{x_{i}} q\right)_{k}=\frac{(q)_{n k}}{(q)_{k-1}^{n}\left(1-q^{k}\right) \cdots\left(1-q^{n k}\right)} \sum_{S} \pm\left(-q^{k}\right)^{|S|} f_{S}\left(q^{k}, q\right) .
$$

The desired rational function is then given by

$$
R_{n}\left(q^{k}, q ; \alpha\right)=\frac{\left(1-q^{k}\right)^{n}}{\left(1-q^{k}\right)\left(1-q^{2 k}\right) \cdots\left(1-q^{n k}\right)} \sum_{S} \pm\left(-q^{k}\right)^{|S|} f_{S}\left(q^{k}, q\right) .
$$

Proof of Lemma 2.2. When regarded as Laurent series in $x_{0}$, the equality

$$
\left(\frac{x_{0}}{x_{i}}\right)_{a}\left(\frac{x_{i}}{x_{0}} q\right)_{b}=q^{\left(\begin{array}{c}
b+1 \\
2
\end{array}\right)}\left(-\frac{x_{i}}{x_{0}}\right)^{b}\left(\frac{x_{0}}{x_{i}} q^{-b}\right)_{a+b}
$$


can be easily shown to hold for all integers $a$. Rewrite $M_{n}^{\prime}(a, b, k, m, l ; q)$ as

$$
\left.\mathrm{CT} \prod_{i=1}^{n} q^{\left(b_{i}^{*}+1\right.}\right)\left(-\frac{x_{i}}{x_{0}}\right)^{b_{i}^{*}+\chi(i \leqslant m)}\left(\frac{x_{0}}{x_{i}} q^{-b_{i}^{*}}\right)_{a+b_{i}^{*}} \prod_{1 \leqslant i<j \leqslant n}\left(\frac{x_{i}}{x_{j}}\right)_{k}\left(\frac{x_{j}}{x_{i}} q\right)_{k},
$$

where $b_{i}^{*}=b+\chi(i \geqslant n-l+1)$.

The well-known $q$-binomial theorem [2, Theorem 2.1] is the identity

$$
\frac{(b z)_{\infty}}{(z)_{\infty}}=\sum_{k=0}^{\infty} \frac{(b)_{k}}{(q)_{k}} z^{k}
$$

Setting $z=u q^{n}$ and $b=q^{-n}$ in (3.6), we obtain

$$
(u)_{n}=\frac{(u)_{\infty}}{\left(u q^{n}\right)_{\infty}}=\sum_{k=0}^{\infty} q^{k(k-1) / 2}\left[\begin{array}{l}
n \\
k
\end{array}\right](-u)^{k}
$$

for all integers $n$, where $\left[\begin{array}{l}n \\ k\end{array}\right]=\frac{(q)_{n}}{(q)_{k}(q)_{n-k}}$ is the $q$-binomial coefficient.

Using (3.7), we see that for $1 \leqslant i \leqslant n$,

$$
q^{\left(\begin{array}{c}
b_{i}^{*}+1 \\
2
\end{array}\right)}\left(-\frac{x_{i}}{x_{0}}\right)^{b_{i}^{*}+\chi(i \leqslant m)}\left(\frac{x_{0}}{x_{i}} q^{-b_{i}^{*}}\right)_{a+b_{i}^{*}}=\sum_{k_{i} \geqslant 0} C\left(k_{i}\right)\left[\begin{array}{c}
a+b_{i}^{*} \\
k_{i}
\end{array}\right] x_{0}^{k_{i}-b_{i}^{*}-\chi(i \leqslant m)} x_{i}^{b_{i}^{*}+\chi(i \leqslant m)-k_{i}},
$$

where $C\left(k_{i}\right)=(-1)^{k_{i}+b_{i}^{*}+\chi(i \leqslant m)} q^{\left(\begin{array}{c}b_{i}^{*}+1 \\ 2\end{array}\right)+\left(\begin{array}{c}k_{i} \\ 2\end{array}\right)-k_{i} b_{i}^{*}}$.

Expanding the first product in (3.5) and taking constant term in $x_{0}$, we see that, by Proposition 3.1, $M_{n}^{\prime}(a, b, k, m, l ; q)$ becomes

$$
\sum_{\mathbf{k}} \prod_{i=1}^{n}\left[\begin{array}{c}
a+b_{i}^{*} \\
k_{i}
\end{array}\right] \underset{x_{1}, \ldots, x_{n}}{\mathrm{CT}} L\left(x_{1}, \ldots, x_{n} ; \mathbf{k}\right)=\frac{(q)_{n k}}{(q)_{k}^{n}} \sum_{\mathbf{k}} \prod_{i=1}^{n}\left[\begin{array}{c}
a+b_{i}^{*} \\
k_{i}
\end{array}\right] R_{n}\left(q^{k}, q ; \mathbf{k}\right),
$$

for some rational functions $R_{n}\left(q^{k}, q ; \mathbf{k}\right)$ in $q^{k}$ and $q$, where

$$
\begin{aligned}
L\left(x_{1}, \ldots, x_{n} ; \mathbf{k}\right)= & q^{(n-l)\left(\begin{array}{c}
b+1 \\
2
\end{array}\right)+l\left(\begin{array}{c}
b+2 \\
2
\end{array}\right)+\sum_{i=1}^{n}\left(\begin{array}{c}
k_{i} \\
2
\end{array}\right)-b \sum_{i=1}^{n-l} k_{i}-(b+1) \sum_{i=n-l+1}^{n} k_{i}} \\
& \cdot \prod_{i=1}^{m} x_{i}^{b_{i}^{*}+1-k_{i}} \prod_{i=m+1}^{n} x_{i}^{b_{i}^{*}-k_{i}} \prod_{1 \leqslant i<j \leqslant n}\left(\frac{x_{i}}{x_{j}}\right)_{k}\left(\frac{x_{j}}{x_{i}} q\right)_{k}
\end{aligned}
$$

is a Laurent polynomial in $x_{1}, \ldots, x_{n}$ independent of $a$ and the sum ranges over all sequences $\mathbf{k}=\left(k_{1}, \ldots, k_{n}\right)$ of nonnegative integers satisfying $k_{1}+k_{2}+\cdots+k_{n}=n b+m+l$. Since $\left[\begin{array}{c}a+b_{i}^{*} \\ k_{i}\end{array}\right]$ is a polynomial in $q^{a}$ of degree $k_{i}$, each summand in (3.8) is a polynomial in $q^{a}$ of degree at most $k_{1}+k_{2}+\cdots+k_{n}=n b+m+l$, and so is the sum.

The coefficients of $M_{n}^{\prime}(a, b, k, m, l ; q)(q)_{k}^{n} /(q)_{n k}$ in $q^{a}$ are clearly rational functions in $q^{k}$ and $q$. 


\section{Proof of the vanishing lemma}

We will follow notations in $[9,16]$, where different versions of the vanishing lemma were proposed for dealing with $q$-Dyson related constant terms. The new vanishing lemma will be handled by the same idea but we have to carry out the details. We will include some basic ingredients for readers' convenience.

In this section, we let $K=\mathbb{C}(q)$, and assume that all series are in the field of iterated Laurent series $K\left(\left(x_{n}\right)\right)\left(\left(x_{n-1}\right)\right) \cdots\left(\left(x_{0}\right)\right)$. The reason for choosing $K\left(\left(x_{n}\right)\right)\left(\left(x_{n-1}\right)\right) \cdots\left(\left(x_{0}\right)\right)$ as a working field has been explained in [9].

We emphasize that the field $K\left(\left(x_{n}\right)\right)\left(\left(x_{n-1}\right)\right) \cdots\left(\left(x_{0}\right)\right)$ include the field of rational functions as a subfield, so that every rational function is identified with its unique iterated Laurent series expansion. The series expansions of $1 /\left(1-q^{k} x_{i} / x_{j}\right)$ will be especially important.

$$
\begin{aligned}
& \frac{1}{1-q^{k} x_{i} / x_{j}}=\sum_{l=0}^{\infty} q^{k l} x_{i}^{l} x_{j}^{-l}, \text { if } i<j, \\
& \frac{1}{1-q^{k} x_{i} / x_{j}}=\frac{1}{-q^{k} x_{i} / x_{j}\left(1-q^{-k} x_{j} / x_{i}\right)}=\sum_{l=0}^{\infty}-q^{-k(l+1)} x_{i}^{-l-1} x_{j}^{l+1}, \text { if } i>j .
\end{aligned}
$$

The constant term of the series $F(\mathbf{x})$ in $x_{i}$, denoted by $\mathrm{CT}_{x_{i}} F(\mathbf{x})$, is defined to be the sum of those terms in $F(\mathbf{x})$ that are free of $x_{i}$. It follows that

$$
\mathrm{CT}_{x_{i}} \frac{1}{1-q^{k} x_{i} / x_{j}}= \begin{cases}1, & \text { if } i<j \\ 0, & \text { if } i>j .\end{cases}
$$

We shall call the monomial $M=q^{k} x_{i} / x_{j}$ small if $i<j$ and large if $i>j$. Thus the constant term in $x_{i}$ of $1 /(1-M)$ is 1 if $M$ is small and 0 if $M$ is large.

Constant term operators defined in this way has the important commutativity property:

$$
\underset{x_{i}}{\mathrm{CT}} \underset{x_{j}}{\mathrm{CT}} F(\mathbf{x})=\underset{x_{j}}{\mathrm{CT}} \underset{x_{i}}{\mathrm{CT}} F(\mathbf{x})
$$

The degree of a rational function of $x$ is the degree in $x$ of the numerator minus the degree in $x$ of the denominator. For example, if $i \neq j$ then the degree of $1-x_{j} / x_{i}=$ $\left(x_{i}-x_{j}\right) / x_{i}$ is 0 in $x_{i}$ and 1 in $x_{j}$. A rational function is called proper (resp. almost proper) in $x$ if its degree in $x$ is negative (resp. zero).

Let

$$
F=\frac{p\left(x_{k}\right)}{x_{k}^{d} \prod_{i=1}^{m}\left(1-x_{k} / \alpha_{i}\right)}
$$

be a rational function of $x_{k}$, where $p\left(x_{k}\right)$ is a polynomial in $x_{k}$, and the $\alpha_{i}$ are distinct monomials, each of the form $x_{t} q^{s}$. Then the partial fraction decomposition of $F$ with respect to $x_{k}$ has the following form:

$$
F=p_{0}\left(x_{k}\right)+\frac{p_{1}\left(x_{k}\right)}{x_{k}^{d}}+\left.\sum_{j=1}^{m} \frac{1}{1-x_{k} / \alpha_{j}}\left(\frac{p\left(x_{k}\right)}{x_{k}^{d} \prod_{i=1, i \neq j}^{m}\left(1-x_{k} / \alpha_{i}\right)}\right)\right|_{x_{k}=\alpha_{j}},
$$


where $p_{0}\left(x_{k}\right)$ is a polynomial in $x_{k}$, and $p_{1}\left(x_{k}\right)$ is a polynomial in $x_{k}$ of degree less than $d$.

The following lemma has appeared in [16].

Lemma 4.1. Let $F$ be as in (4.2) and (4.3). Then

$$
\underset{x_{k}}{\mathrm{CT}} F=p_{0}(0)+\left.\sum_{j}\left(F\left(1-x_{k} / \alpha_{j}\right)\right)\right|_{x_{k}=\alpha_{j}},
$$

where the sum ranges over all $j$ such that $x_{k} / \alpha_{j}$ is small. In particular, if $F$ is proper in $x_{k}$, then $p_{0}=0$; if $F$ is almost proper in $x_{k}$, then $p_{0}\left(x_{k}\right)=(-1)^{m} \prod_{i=1}^{m} \alpha_{i} \operatorname{LC}_{x_{k}} p\left(x_{k}\right)$, where $\mathrm{LC}_{x_{k}}$ means to take the leading coefficient with respect to $x_{k}$.

The following lemma plays an important role in our argument.

Lemma 4.2. Let $k, b$ and $k_{1}, \ldots, k_{s}$ be nonnegative integers. Then for any $k_{1}, \ldots, k_{s}$ with $0 \leqslant k_{i} \leqslant(s-1) k+b+1$ for all $i$, either $0 \leqslant k_{i} \leqslant b$ for some $i$, or $1-k \leqslant k_{j}-k_{i} \leqslant k$ for some $i<j$, except only when $k_{i}=(s-i) k+b+1$ for $i=1, \ldots, s$.

Proof. Assume $k_{1}, \ldots, k_{s}$ to satisfy that for all $i, b<k_{i} \leqslant(s-1) k+b+1$, and for all $i<j$, either $k_{j}-k_{i}>k$ or $k_{j}-k_{i} \leqslant-k$. Then we need to show that $k_{i}=(s-i) k+b+1$ for $i=1, \ldots, s$.

Let $[b, c]$ denote the set $\{b, b+1, \ldots, c\}$ for integers $b \leqslant c$. Under the hypothesis of the first paragraph, the integers $k_{i}$ are $s$ points at distance at least $k$ in an interval of length $(s-1) k$, namely $[b+1, b+1+(s-1) k]$. Thus, there exists some permutation $\sigma$ such that $k_{\sigma(i)}=b+1+(s-i) k$. For $i=1, \ldots, s-1$, we have $k_{\sigma(i+1)}-k_{\sigma(i)}=-k$, which implies $\sigma(i+1)>\sigma(i)$. This can only occur when $\sigma$ is the identity permutation.

Let

$$
\begin{aligned}
Q(h) & =\prod_{i=1}^{m} \frac{\left(x_{i} / x_{0}\right)_{b+1+\chi(i \geqslant n-l+1)}}{\left(1-x_{0} / x_{i}\right) \cdots\left(1-x_{0} /\left(x_{i} q^{h}\right)\right)} \prod_{i=m+1}^{n} \frac{\left(x_{i} q / x_{0}\right)_{b+\chi(i \geqslant n-l+1)}}{\left(1-x_{0} /\left(x_{i} q\right)\right) \cdots\left(1-x_{0} /\left(x_{i} q^{h}\right)\right)} \\
& \cdot \prod_{1 \leqslant i<j \leqslant n}\left(\frac{x_{i}}{x_{j}}\right)_{k}\left(\frac{x_{j}}{x_{i}} q\right)_{k} .
\end{aligned}
$$

By Lemma 2.2, we know that $M_{n}^{\prime}(a, b, k, m, l ; q)$ is a polynomial in $q^{a}$, so the definition of $a$ can be extended to negative integers. Then, since $(u)_{n}=(u)_{\infty} /\left(u q^{n}\right)_{\infty}$ for all integers $n$, we have

$$
M_{n}^{\prime}(-h, b, k, m, l ; q)=\underset{\mathbf{x}}{\mathrm{CT}} Q(h) .
$$

The vanishing lemma says that $\mathrm{CT}_{\mathbf{x}} Q(h)=0$ for every $h$ in $(2.2)$.

We attack the vanishing lemma by repeated application of Lemma 4.1. This will give a big sum of terms, each will be detected to be 0 by Lemma 4.2. This is better summarized in the following Lemma 4.3. To state the lemma, we need more notations. 
For any rational function $F$ of $x_{0}, x_{1}, \ldots, x_{n}$, and for sequences of integers $k=$ $\left(k_{1}, k_{2}, \ldots, k_{s}\right)$ and $r=\left(r_{1}, r_{2}, \ldots, r_{s}\right)$ let $E_{\mathbf{r}, \mathbf{k}} F$ be the result of replacing $x_{r_{i}}$ in $F$ with $x_{r_{s}} q^{k_{s}-k_{i}}$ for $i=0,1, \ldots, s-1$, where we set $r_{0}=k_{0}=0$. Then for $0<r_{1}<r_{2}<\ldots<$ $r_{s} \leqslant n$ and $0 \leqslant k_{i} \leqslant h$, we define

$$
Q(h \mid \mathbf{r} ; \mathbf{k})=Q\left(h \mid r_{1}, \ldots, r_{s} ; k_{1}, \ldots, k_{s}\right)=E_{\mathbf{r}, \mathbf{k}}\left[Q(h) \prod_{i=1}^{s}\left(1-\frac{x_{0}}{x_{r_{i}} q^{k_{i}}}\right)\right] .
$$

Note that the product on the right hand side of (4.6) cancels all the factors in the denominator of $Q$ that would be taken to zero by $E_{\mathbf{r}, \mathbf{k}}$. If $k_{i}=0$ for some $i$ and $r_{i} \leqslant m$, then $Q(h \mid \mathbf{r} ; \mathbf{k})$ has the factor $E_{\mathbf{r}, \mathbf{k}}\left[\left(x_{r_{i}} / x_{0}\right)_{b+1+\chi\left(r_{i} \geqslant n-l+1\right)}\right]=0$. If $k_{i}=0$ for some $i$ and $r_{i}>m$, by the definition of $Q(h \mid \mathbf{r} ; \mathbf{k})$ in (4.6), the factor $1-x_{0} / x_{r_{i}}$ appears in $Q(h \mid \mathbf{r} ; \mathbf{k})$, but it cancels nothing in the denominator of $Q(h)$. Thus it would be taken to zero by $E_{\mathbf{r}, \mathbf{k}}$ and $Q(h \mid \mathbf{r} ; \mathbf{k})=0$. Therefore, if $k_{i}=0$ for some $i$, then $Q(h \mid \mathbf{r} ; \mathbf{k})=0$.

As a warm up, it is easy to check that $Q(h)$ is proper in $x_{0}$ with degree $-n h-m$. Thus applying Lemma 4.1 gives

$$
\mathrm{CT}_{x_{0}} Q(h)=\sum_{\substack{1 \leqslant r_{1,1} \leqslant m \\ 0 \leqslant k_{1,1} \leqslant h}} Q\left(h \mid r_{1,1} ; k_{1,1}\right)+\sum_{\substack{m+1 \leqslant r_{2,1} \leqslant n \\ 1 \leqslant k_{2,1} \leqslant h}} Q\left(h \mid r_{2,1} ; k_{2,1}\right) .
$$

Since $Q\left(h \mid r_{1,1} ; 0\right)=0$, we can rewrite (4.7) as

$$
\mathrm{CT}_{x_{0}} Q(h)=\sum_{\substack{1 \leqslant r_{1} \leqslant n \\ 1 \leqslant k_{1} \leqslant h}} Q\left(h \mid r_{1} ; k_{1}\right) .
$$

This formula is compatible with the following lemma if we treat $Q(h)=Q(h \mid \varnothing ; \varnothing)$.

Lemma 4.3. The rational functions $Q(h \mid \mathbf{r} ; \mathbf{k})$ have the following two properties:

(i) If $0 \leqslant k_{i} \leqslant(s-1) k+b+\chi(s \geqslant n-l+1)$ for all $i$ with $1 \leqslant i \leqslant s$, then $Q(h \mid \mathbf{r} ; \mathbf{k})=0$.

(ii) Suppose $k>b+1$ and $h \in D_{1} \bigcup D_{2} \bigcup D_{3} \bigcup\{(n-l-1) k+b+1\}$. If $k_{i}>(s-1) k+$ $b+\chi(s \geqslant n-l+1)$ for some $i$ with $1 \leqslant i \leqslant s$ and $n>s$, then

$$
\underset{x_{r_{s}}}{\mathrm{CT}} Q(h \mid \mathbf{r} ; \mathbf{k})=\sum_{\substack{r_{s}<r_{s+1} \leqslant n \\ 0 \leqslant k_{s+1} \leqslant h}} Q\left(h \mid r_{1}, \ldots, r_{s}, r_{s+1} ; k_{1}, \ldots, k_{s}, k_{s+1}\right) .
$$

Proof of property (i). By Lemma 4.2, if $0 \leqslant k_{i} \leqslant(s-1) k+b+\chi(s \geqslant n-l+1)$ for all $i$, the $k_{i}$ 's have to be in one of the following three cases. Case 1: $0 \leqslant k_{i} \leqslant b$ for some $1 \leqslant i \leqslant s$; Case $2: 1-k \leqslant k_{j}-k_{i} \leqslant k$ for some $i<j$; Case $3: k_{i}=(s-i) k+b+1$ for $i=1, \ldots, s$. Note that Case 3 occurs only when $s \geqslant n-l+1$.

Case 1: $0 \leqslant k_{i} \leqslant b$ for some $1 \leqslant i \leqslant s$. If $r_{i} \leqslant m$, then $Q(h \mid \mathbf{r} ; \mathbf{k})$ has the factor

$$
E_{\mathbf{r}, \mathbf{k}}\left[\left(\frac{x_{r_{i}}}{x_{0}}\right)_{b+1+\chi\left(r_{i} \geqslant n-l+1\right)}\right]=\left(\frac{x_{r_{s}} q^{k_{s}-k_{i}}}{x_{r_{s}} q^{k_{s}}}\right)_{b+1+\chi\left(r_{i} \geqslant n-l+1\right)}=\left(q^{-k_{i}}\right)_{b+1+\chi\left(r_{i} \geqslant n-l+1\right)}=0 .
$$


If $r_{i}>m$, then $Q(h \mid \mathbf{r} ; \mathbf{k})=0$ for $k_{i}=0$ and for $1 \leqslant k_{i} \leqslant b$ it has the factor

$$
E_{\mathbf{r}, \mathbf{k}}\left[\left(\frac{x_{r_{i}} q}{x_{0}}\right)_{b+\chi\left(r_{i} \geqslant n-l+1\right)}\right]=\left(\frac{x_{r_{s}} q^{1+k_{s}-k_{i}}}{x_{r_{s}} q^{k_{s}}}\right)_{b+\chi\left(r_{i} \geqslant n-l+1\right)}=\left(q^{1-k_{i}}\right)_{b+\chi\left(r_{i} \geqslant n-l+1\right)}=0 .
$$

Case 2: $1-k \leqslant k_{j}-k_{i} \leqslant k$ for some $i<j$. In this case $Q(h \mid \mathbf{r} ; \mathbf{k})$ has the factor

$$
E_{\mathbf{r}, \mathbf{k}}\left[\left(\frac{x_{r_{i}}}{x_{r_{j}}}\right)_{k}\left(\frac{x_{r_{j}}}{x_{r_{i}}} q\right)_{k}\right]
$$

which is equal to

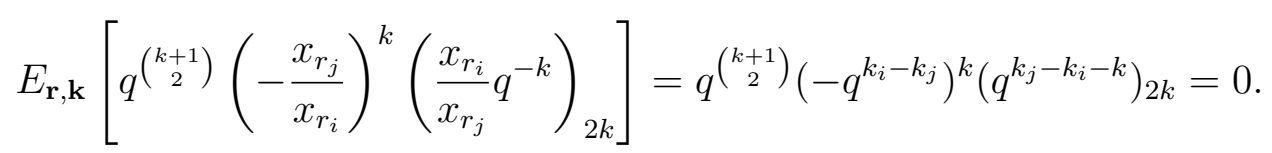

Case 3: $k_{i}=(s-i) k+b+1$ for $i=1, \ldots, s$. In this case we only need the value of $k_{s}$. Since Case 3 only occurs when $s \geqslant n-l+1$, we have $r_{s} \geqslant s \geqslant n-l+1$. If $r_{s} \leqslant m$, then $Q(h \mid \mathbf{r} ; \mathbf{k})$ has the factor

$$
E_{\mathbf{r}, \mathbf{k}}\left[\left(\frac{x_{r_{s}}}{x_{0}}\right)_{b+1+\chi\left(r_{s} \geqslant n-l+1\right)}\right]=E_{\mathbf{r}, \mathbf{k}}\left[\left(\frac{x_{r_{s}}}{x_{0}}\right)_{b+2}\right]=\left(\frac{x_{r_{s}}}{x_{r_{s}} q^{k_{s}}}\right)_{b+2}=\left(q^{-b-1}\right)_{b+2}=0 .
$$

If $r_{s}>m$, then $Q(h \mid \mathbf{r} ; \mathbf{k})$ has the factor

$$
E_{\mathbf{r}, \mathbf{k}}\left[\left(\frac{x_{r_{s}} q}{x_{0}}\right)_{b+\chi\left(r_{s} \geqslant n-l+1\right)}\right]=E_{\mathbf{r}, \mathbf{k}}\left[\left(\frac{x_{r_{s}} q}{x_{0}}\right)_{b+1}\right]=\left(\frac{x_{r_{s}} q}{x_{r_{s}} q^{k_{s}}}\right)_{b+1}=\left(q^{-b}\right)_{b+1}=0 .
$$

Proof of property (ii). Note that since $h \geqslant k_{i}$ for all $i$ and $h \in D_{1} \bigcup D_{2} \bigcup D_{3} \bigcup\{(n-$ $l-1) k+b+1\}$, the hypothesis implies that $h>s k-\chi(s<m)$.

We only show that $Q(h \mid \mathbf{r} ; \mathbf{k})$ is proper in $x_{r_{s}}$ so that Lemma 4.1 applies. The rest is the same as that in the proof of [9, Lemma 5.1]. To this end we write $Q(h \mid \mathbf{r} ; \mathbf{k})$ as $N / D$, in which $N$ (the numerator) is

$$
E_{\mathbf{r} ; \mathbf{k}}\left[\prod_{i=1}^{m}\left(\frac{x_{i}}{x_{0}}\right)_{b+1+\chi(i \geqslant n-l+1)} \prod_{i=m+1}^{n}\left(\frac{x_{i} q}{x_{0}}\right)_{b+\chi(i \geqslant n-l+1)} \cdot \prod_{\substack{1 \leqslant i, j \leqslant n \\ i \neq j}}\left(\frac{x_{i}}{x_{j}} q^{\chi(i>j)}\right)_{k}\right],
$$

and $D$ (the denominator) is

$$
E_{\mathbf{r} ; \mathbf{k}}\left[\prod_{i=1}^{m}\left(\frac{x_{0}}{x_{i} q^{h}}\right)_{h+1} \prod_{i=m+1}^{n}\left(\frac{x_{0}}{x_{i} q^{h}}\right)_{h} / \prod_{i=1}^{s}\left(1-\frac{x_{0}}{x_{r_{i}} q^{k_{i}}}\right)\right] .
$$

Now let $R=\left\{r_{0}, r_{1}, \ldots, r_{s}\right\}$. Then the degree in $x_{r_{s}}$ of

$$
E_{\mathbf{r} ; \mathbf{k}}\left[\left(1-\frac{x_{i}}{x_{j}} q^{l}\right)\right]
$$


is 1 if $i \in R$ and $j \notin R$, and is 0 otherwise, as is easily seen by checking the four cases. Thus the part of $N$ contributing to the degree in $x_{r_{s}}$ is

$$
E_{\mathbf{r} ; \mathbf{k}}\left[\prod_{i=1}^{s} \prod_{j \neq r_{0}, \ldots, r_{s}}\left(\frac{x_{r_{i}}}{x_{j}} q^{\chi\left(r_{i}>j\right)}\right)_{k}\right]
$$

which has degree $(n-s) s k$, and the part of $D$ contributing to the degree in $x_{r_{s}}$ is

$$
E_{\mathbf{r} ; \mathbf{k}}\left[\prod_{\substack{j \neq r_{0}, \ldots, r_{s} \\ j \leqslant m}}\left(\frac{x_{0}}{x_{j} q^{h}}\right)_{h+1} \prod_{\substack{j \neq r_{0}, \ldots, r_{s} \\ j>m}}\left(\frac{x_{0}}{x_{j} q^{h}}\right)_{h}\right],
$$

which has degree at least $(n-s) h+\chi(s<m)$.

Thus the total degree of $Q(h \mid \mathbf{r} ; \mathbf{k})$ in $x_{r_{s}}$ is at most

$$
(n-s)(s k-h)-\chi(s<m) \leqslant(n-s)(\chi(s<m)-1)-\chi(s<m)<0,
$$

so $Q(h \mid \mathbf{r} ; \mathbf{k})$ is proper in $x_{r_{s}}$.

Now we are ready to prove the vanishing lemma.

Proof of the vanishing lemma. Recall that $\mathrm{CT}_{\mathbf{x}} Q(h)=M_{n}^{\prime}(-a, b, k, m, l ; q)$. We prove by induction on $n-s$ that

$$
\underset{\mathbf{x}}{\mathrm{CT}} Q(h \mid \mathbf{r} ; \mathbf{k})=0
$$

the lemma is the case $s=0$. (Note that taking the constant term with respect to a variable that does not appear has no effect.) We may assume that $s \leqslant n$ and $0<r_{1}<\cdots<r_{s} \leqslant n$, since otherwise $Q(h \mid \mathbf{r} ; \mathbf{k})$ is not defined. If $s=n$ then $r_{i}$ must equal $i$ for $i=1, \ldots, n$ and thus $Q(h \mid \mathbf{r} ; \mathbf{k})=Q\left(h \mid 1,2, \ldots, n ; k_{1}, k_{2}, \ldots, k_{n}\right)$, which by property (i) of Lemma 4.3 is 0 , since for each $i, k_{i} \leqslant h \leqslant(n-1) k+b+\chi(l>0)$. Now suppose that $0 \leqslant s<n$. Applying $\mathrm{CT}_{\mathbf{x}}$ to both sides of (4.9) gives

$$
\underset{\mathbf{x}}{\mathrm{CT}} Q(h \mid \mathbf{r} ; \mathbf{k})=\sum_{\substack{r_{s}<r_{s+1} \leqslant n \\ 0 \leqslant k_{s+1} \leqslant h}} \mathrm{CT}_{\mathbf{x}} Q\left(h \mid r_{1}, \ldots, r_{s}, r_{s+1} ; k_{1}, \ldots, k_{s}, k_{s+1}\right)
$$

when property (ii) of Lemma 4.3 applies. Thus by Lemma $4.3, \mathrm{CT}_{\mathbf{x}} Q(h \mid \mathbf{r} ; \mathbf{k})$ is either 0 or is a sum of terms, all of which are 0 by induction.

\section{Proof for the extra point}

We need the following lemma.

Lemma 5.1. Assume Theorem 1.3 holds for smaller values of $n$. Let $h=(n-l-1) k+b+1$. If $0 \leqslant m, l<n$ and $k>b+1$ then

$$
\mathrm{CT}_{x} Q(h \mid 1, \ldots, n-l ; h, h-k \ldots, b+1)=M_{n}(-h, b, k, m, l ; q) .
$$


Proof. We have to split into the following two cases.

Case 1: $m+l \leqslant n$. Then

$$
Q(h \mid 1, \ldots, n-l ; h, h-k, \ldots, b+1)=D \cdot(A \cdot B) \cdot \prod_{n-l+1 \leqslant i<j \leqslant n}\left(\frac{x_{i}}{x_{j}}\right)_{k}\left(\frac{x_{j}}{x_{i}} q\right)_{k},
$$

where

$$
\begin{gathered}
D=\prod_{i=1}^{m} \frac{\left(q^{-(n-l-i) k-b-1}\right)_{b+1}}{(q)_{(n-l-i) k+b+1}\left(q^{-(i-1) k}\right)_{(i-1) k}} \prod_{i=m+1}^{n-l} \frac{\left(q^{-(n-l-i) k-b}\right)_{b}}{(q)_{(n-l-i) k+b}\left(q^{-(i-1) k}\right)_{(i-1) k}} \\
\quad \times \prod_{1 \leqslant i<j \leqslant n-l}\left(q^{(i-j) k}\right)_{k}\left(q^{(j-i) k+1}\right)_{k} \\
=\prod_{i=1}^{m} \frac{\left(q^{-(n-l-i) k-b-1}\right)_{b+1}}{(q)_{(n-l-i) k+b+1}} \prod_{i=m+1}^{n-l} \frac{\left(q^{-(n-l-i) k-b}\right)_{b}}{(q)_{(n-l-i) k+b}} \prod_{j=1}^{n-l} \frac{\prod_{i=1}^{j-1}\left(q^{(i-j) k}\right)_{k}\left(q^{(j-i) k+1}\right)_{k}}{\left(q^{-(j-1) k}\right)_{(j-1) k}} \\
=\prod_{i=1}^{m} \frac{\left(q^{-(n-l-i) k-b-1}\right)_{b+1}}{(q)_{(n-l-i) k+b+1}} \prod_{i=m+1}^{n-l} \frac{\left(q^{-(n-l-i) k-b}\right)_{b}}{(q)_{(n-l-i) k+b}} \prod_{j=1}^{n-l} \frac{(q)_{j k}}{(q)_{k}}, \\
A=\prod_{i=n-l+1}^{n} \frac{\left(\frac{x_{i}}{x_{n-l}} q^{-b}\right)_{b+1}}{\left(\frac{x_{n-l}}{x_{i}} q^{-(n-l-1) k}\right)_{(n-l-1) k+b+1}}
\end{gathered}
$$

and

$$
\begin{aligned}
B & =\prod_{\substack{1 \leqslant i \leqslant n-l \\
n-l+1 \leqslant j \leqslant n}}\left(\frac{x_{n-l}}{x_{j}} q^{-(n-l-i) k}\right)_{k}\left(\frac{x_{j}}{x_{n-l}} q^{(n-l-i) k+1}\right)_{k} \\
& =\prod_{j=n-l+1}^{n}\left(\frac{x_{n-l}}{x_{j}} q^{-(n-l-1) k}\right)_{(n-l) k}\left(\frac{x_{j}}{x_{n-l}} q\right)_{(n-l) k} .
\end{aligned}
$$

For $k>b+1$, after cancelations and combinations, we obtain

$$
\begin{aligned}
& \mathrm{CT} A \cdot B \cdot \prod_{n-l+1 \leqslant i<j \leqslant n}\left(\frac{x_{i}}{x_{j}}\right)_{k}\left(\frac{x_{j}}{x_{i}} q\right)_{k} \\
= & \mathrm{CT}_{x} \prod_{i=n-l+1}^{n}\left(\frac{x_{n-l}}{x_{i}} q^{b+1}\right)_{k-b-1}\left(\frac{x_{i}}{x_{n-l}} q^{-b}\right)_{(n-l) k+b+1} \prod_{n-l+1 \leqslant i<j \leqslant n}\left(\frac{x_{i}}{x_{j}}\right)_{k}\left(\frac{x_{j}}{x_{i}} q\right)_{k} \\
= & \mathrm{CT}_{x} \prod_{i=n-l+1}^{n}\left(\frac{x_{n-l}}{x_{i}}\right)_{k-b-1}\left(\frac{x_{i}}{x_{n-l}} q\right)_{(n-l) k+b+1} \prod_{n-l+1 \leqslant i<j \leqslant n}\left(\frac{x_{i}}{x_{j}}\right)_{k}\left(\frac{x_{j}}{x_{i}}\right)_{k},
\end{aligned}
$$


where the last equality is obtained by making the substitution $x_{n-l}=x_{n-l} q^{-b-1}$. This is just $M_{l}^{\prime}(k-b-1,(n-l) k+b+1, k, 0,0 ; q)$. By Remark 2.4 (or the hypothesis), we obtain

$$
\mathrm{CT} Q(h)=D \cdot \prod_{i=0}^{l-1} \frac{(q)_{(n-l+i+1) k}(q)_{(i+1) k}}{(q)_{(i+1) k-b-1}(q)_{(n-l+i) k+b+1}(q)_{k}},
$$

which can be routinely checked to be equal to $M_{n}(-h, b, k, m, l ; q)$.

Case 2: $m+l>n$. The computation is similar to but more complicated than case 1 . Indeed we need the case 1 result in some sense. We omit some details for brevity. We have

$$
Q(h \mid 1, \ldots, n-l ; h, h-k, \ldots, b+1)=D^{\prime} \cdot\left(A^{\prime} \cdot B^{\prime}\right) \cdot \prod_{n-l+1 \leqslant i<j \leqslant n}\left(\frac{x_{i}}{x_{j}}\right)_{k}\left(\frac{x_{j}}{x_{i}} q\right)_{k},
$$

where

$$
\begin{aligned}
D^{\prime} & =\prod_{i=1}^{n-l} \frac{\left(q^{-(n-l-i) k-b-1}\right)_{b+1}}{(q)_{(n-l-i) k+b+1}\left(q^{-(i-1) k}\right)_{(i-1) k}} \cdot \prod_{1 \leqslant i<j \leqslant n-l}\left(q^{(i-j) k}\right)_{k}\left(q^{(j-i) k+1}\right)_{k} \\
& =\prod_{i=1}^{n-l} \frac{\left(q^{-(n-l-i) k-b-1}\right)_{b+1}(q)_{i k}}{(q)_{(n-l-i) k+b+1}(q)_{k}}
\end{aligned}
$$

and $A^{\prime}$ and $B^{\prime}$ are similar to $A$ and $B$, with $A^{\prime} B^{\prime}$ simplifies as

$$
\begin{aligned}
A^{\prime} \cdot B^{\prime}= & \prod_{i=n-l+1}^{m}\left(\frac{x_{n-l}}{x_{i}} q^{b+2}\right)_{k-b-2}\left(\frac{x_{i}}{x_{n-l}} q^{-b-1}\right)_{(n-l) k+b+2} \\
& \times \prod_{i=m+1}^{n}\left(\frac{x_{n-l}}{x_{i}} q^{b+1}\right)_{k-b-1}\left(\frac{x_{i}}{x_{n-l}} q^{-b}\right)_{(n-l) k+b+1} .
\end{aligned}
$$

A similar computation gives

$$
\begin{aligned}
\mathrm{CT} A^{\prime} \cdot B^{\prime} \cdot \prod_{n-l+1 \leqslant i<j \leqslant n}\left(\frac{x_{i}}{x_{j}}\right)_{k}\left(\frac{x_{j}}{x_{i}} q\right)_{k} \\
\quad=M_{l}^{\prime}(k-b-1,(n-l) k+b+1, k, m-n+l, 0 ; q),
\end{aligned}
$$

which is the constant term in (1.3) in case 1 , and is known to be $M_{l}(k-b-1,(n-l) k+$ $b+1, k, m-n+l, 0 ; q)$ by the hypothesis. Then it only left to show that

$$
D^{\prime} \cdot \prod_{i=0}^{l-1} \frac{(q)_{(n-l+i+1) k}(q)_{(i+1) k}}{(q)_{(i+1) k-b-1-\chi(i<m-n+l)}(q)_{(n-l+i) k+b+1+\chi(i \geqslant n-m)}(q)_{k}}=M_{n}(-h, b, k, m, l ; q),
$$

which is routine. 
Note that we can avoid using the induction hypothesis. The truth of Lemma 5.1 in case 1 results in the truth of Theorem 1.3 in case 1, which is needed in the case 2 of Lemma 5.1.

Now we are ready to deal with the extra point.

Proof of Lemma 2.5. As we discussed in (4.8), $\mathrm{CT}_{x_{0}} Q(h)$ can be written as

$$
\underset{x_{0}}{\mathrm{CT}} Q(h)=\sum_{\substack{1 \leqslant r_{1} \leqslant n \\ 1 \leqslant k_{1} \leqslant h}} Q\left(h \mid r_{1}, k_{1}\right) .
$$

Iteratively apply Lemma 4.3 to each summand if applicable. Finally we get

$$
\underset{\mathbf{x}}{\mathrm{CT}} Q(h)=\underset{\mathbf{x}}{\mathrm{CT}} \sum_{r_{1}, \ldots, r_{s}, k_{1}, \ldots, k_{s}} Q\left(h \mid r_{1}, \ldots, r_{s} ; k_{1}, \ldots, k_{s}\right),
$$

where the sum ranges over all the $r$ 's and the $k$ 's with $0<r_{1}<\cdots<r_{s} \leqslant n, 0 \leqslant$ $k_{1}, k_{2}, \ldots, k_{s} \leqslant h$ such that Lemma 4.3 does not apply. Note that we may have different $s$.

Since $h=(n-l-1) k+b+1$ and $0 \leqslant k_{i} \leqslant h$, by Lemma 4.2 , there leaves only one term for which Lemma 4.3 is not applicable. This term corresponds to $k_{i}=(n-l-i) k+b+1$ for $i=1, \ldots, n-l$ and $r_{i}=i$ for $i=1, \ldots, n-l$. It follows that

$$
\mathrm{CT}_{x} Q(h)=\underset{x}{\mathrm{CT}} Q(h \mid 1, \ldots, n-l ; h, h-k, \ldots, b+1) .
$$

The lemma then follows from Lemma 5.1.

The extra point $h=(n-l-1) k+b+1$ in Lemma 2.5 is not easy to find. This $h$ seems to be the only choice of the extra point for which it is not hard to show that $\mathrm{CT}_{x} Q(h)=M_{n}(-h, b, k, m, l ; q)$. Intuitively a desired extra point must be chosen from boundary values, i.e., values next to the vanishing points listed in (2.2).

Firstly, the boundary values $h=(n-l-2) k+b+1,(n-l-3) k+b+1, \ldots, b+1$ do not work. To see this, take $n=3, m=l=1$ for example. Then we can only get

$$
\begin{gathered}
Q(b+1)=(-1)^{b} q^{-\left(\begin{array}{c}
b+1 \\
2
\end{array}\right)} \underset{x}{\mathrm{CT}} \frac{\left(q / x_{1}\right)_{k-b-1}\left(x_{1}\right)_{b+k+1}\left(1 / x_{3}\right)_{k-b-1}\left(x_{3} q\right)_{b+k+1}}{1-1 /\left(x_{1} q^{b+1}\right)}\left(\frac{x_{1}}{x_{3}}\right)_{k}\left(\frac{x_{3}}{x_{1}} q\right)_{k} \\
+(-1)^{b+1} q^{-\left(\begin{array}{c}
b+2 \\
2
\end{array}\right)} \mathrm{CT}_{x} \frac{\left(1 / x_{2}\right)_{k-b-1}\left(x_{2} q\right)_{b+k+1}\left(1 / x_{3}\right)_{k-b-1}\left(x_{3} q\right)_{b+k+1}}{1-x_{2} q^{b+1}} \cdot\left(\frac{x_{2}}{x_{3}}\right)_{k}\left(\frac{x_{3}}{x_{2}} q\right)_{k} .
\end{gathered}
$$

Secondly, the boundary values $h=m k,(m+1) k, \ldots,(n-1) k$ do not work either for a similar reason.

\section{Acknowledgements}

The authors would like to thank Christian Krattenthaler for pointing out a trivial identity in the earlier draft, and the referee for many useful suggestions and comments. This work was supported by the National Science Foundation of China (No. 11171231, 11101435) and the Natural Science Foundation of Hunan Province, China. 


\section{References}

[1] G. E. Andrews. Problems and prospects for basic hypergeometric functions. Theory and application of special functions. Academic Press, New York, 191-224, 1975.

[2] G. E. Andrews. The Theory of Partitions. Cambridge University Press, Cambridge, 1998.

[3] K. Aomoto. Jacobi polynomials associated with Selberg integrals. SIAM J. Math. Anal., 18:545-549, 1987.

[4] T. H. Baker and P. J. Forrester. Generalizations of the $q$-Morris constant term identity. J. Combin. Theory Ser. A, 81:69-87, 1998.

[5] T. Chappell, A. Lascoux, S. Warnaar, and W. Zudilin. Logarithmic and complex constant term identities. Computational and analytical mathematics, Springer Proc. Math. Stat., 50, Springer, New York, 219-250, 2013.

[6] I. Cherednik. Double affine Hecke algebras and Macdonald's conjectures. Ann. Math., 141:191-216, 1995.

[7] F. J. Dyson. Statistical theory of the energy levels of complex systems I. J. Math. Phys., 3:140-156, 1962.

[8] I. M. Gessel, L. Lv, G. Xin, and Y. Zhou. A unified elementary approach to the Dyson, Morris, Aomoto, and Forrester constant terms. J. Combin. Theory Ser. A, 115:1417-1435, 2008.

[9] I. M. Gessel and G. Xin. A short proof of the Zeilberger-Bressoud $q$-Dyson theorem. Proc. Amer. Math. Soc., 134:2179-2187, 2006.

[10] I. J. Good. Short proof of a conjecture by Dyson. J. Math. Phys., 11:1884, 1970.

[11] J. Gunson, unpublised.

[12] L. Habsieger. Une q-intégrale de Selberg et Askey. SIAM J. Math. Anal., 19:14751489, 1988.

[13] K. W. J. Kadell. A proof of Askey's conjectured q-analogue of Selberg's integral and a conjecture of Morris. SIAM J. Math. Anal., 19:969-986, 1988.

[14] G. Károlyi and Z. L. Nagy. A simple proof of the Zeilberger-Bressoud q-Dyson Theorem. Proc. Amer. Math. Soc., 142:3007-3011, 2014.

[15] G. Károlyi, Z. L. Nagy, F. V. Petrov, and V. Volkov. A new approach to constant term identities and Selberg-type integrals. arXiv:1312.6369.

[16] L. Lv, G. Xin, and Y. Zhou. A family of $q$-Dyson style constant term identities. J. Combin. Theory Ser. A, 116:12-29, 2009.

[17] I. G. Macdonald. Some conjectures for root systems. SIAM J. Math. Anal., 13:9881007, 1982.

[18] W. G. Morris. Constant term identities for finite and affine root system. Ph.D. Thesis, University of Wisconsin, Madison, 1982. 
[19] A. Selberg. Bemerkninger om et multipelt integral. Norsk Mat. Tidsskr., 26:71-78, 1944.

[20] J. R. Stembridge. First layer formulas for characters of $S L(n, \mathbb{C})$. Trans. Amer. Math. Soc., 299:319-350, 1987.

[21] K. G. Wilson. Proof of a conjecture by Dyson. J. Math. Phys., 3:1040-1043, 1962.

[22] D. Zeilberger. A combinatorial proof of Dyson's conjecture. Discrete Math., 41:317321, 1982.

[23] D. Zeilberger and D. M. Bressoud. A proof of Andrews' q-Dyson conjecture. Discrete Math., 54:201-224, 1985.

[24] D. Zeilberger. A Stembridge-Stanton style elementary proof of the Habsieger-Kadell q-Morris identity. Discrete Math., 79:313-322, 1990. 